\title{
Research on Computer Network Virtual Laboratory based on ASP.NET
}

\author{
JIA Xuebin ${ }^{1, a}$ \\ ${ }^{1}$ Department of Computer, Wuhan Polytechnic, Wuhan 430074, China \\ ajiaxuebin@126.com
}

Keywords: ASP.NET; Data Storage and Processing; System Performance; System Information.

\begin{abstract}
In this paper, we conduct research on the design and implementation of computer network virtual laboratory based on ASP.NET. Virtual laboratory is a kind of special, distributed problem solving environment which is to provide the users a web-based experiment teaching, technical exchanges, joint research and collaborative work platform which is a kind of virtual prototype system based on computer science research and engineering design. In our system, the necessary structures are discussed with sufficient introduction. The result shows the effectiveness of the approach.
\end{abstract}

\section{Introduction}

A web application is an application that is accessed with a web browser over a network such as the Internet or an intranet. Web applications are popular due to the ubiquity of the browser as a client. The ability to update and maintain web applications without distributing and installing software on potentially thousands of client computers is a key reason for their popularity. With the wide application of computer network, it needs more people to master, management and conduct research and development of computer network. The characteristics of computer network itself determines to understand and study is a difficult process, therefore, beginners have to face the abstract and complex network protocol, even professionals need to use complex network simulation software by writing code for studying the subject [1-2]. Virtual laboratory is a kind of special, distributed problem solving environment, is to provide the users a web-based experiment teaching, technical exchanges, joint research, collaborative work platform, is a kind of virtual prototype system based on computer science research and engineering design method of new, is in addition to theoretical and physical experiment of a third type of research design means and form. The characteristics of remote education are the separation between teachers and students, so the implementation of each teaching link and face-to-face form are very different especially in the experimental teaching. Experiment is the majority of the engineering courses and one of the most important aspects of the application classes, due to lacking of laboratory hands-on opportunities the course teaching effect will be discounted. But in the remote education, experiment teaching organization has a lot of difficulties. First of all, it can't be like ordinary colleges and universities of all kinds of laboratory construction which is also equipped with more hard experiment personnel; Secondly, according to the remote education breakthrough time and space limitations we emphasizes the characteristics of autonomous learning to reduce the cost of running. Therefore, it is impossible to build large laboratory system.

Web applications are used to implement various sorts of applications including E-commerce, online banking, webmail, business applications and many other functions. Virtual laboratory is one of the hottest applications. The so-called virtual experiment, according to the United Nations educational, scientific and cultural organization (UNESCO) is defined as the use of decentralized information and communication technology in scientific research and other creative activities for long cooperation and the experimental process of an electronic collaboration [3]. Virtual laboratory component generally contains the following sections: virtual prototype experiments, application specific information database, connect to the network tool of scientific equipment, mutual cooperation, based on the simulation, data analysis, find and simplify and data visualization software and the demand for network bandwidth. Virtual laboratory is authentication type, test type, design type, type and innovative type of error correction. This paper involved in virtual experiment is the use of computer technology and Internet technology, as a Web for the realization of the platform, a series 
of software and hardware organically, so as to realize the real experiments and experimental results of computer simulation. This kind of virtual experiment based on the real experiment, components, instruments and equipment of experiments using computer simulation, the experimenter through the mouse to click and drag and keyboard operation, can be like the real components of virtual experiment equipment, so as to complete the whole process of virtual experiment. In the figure 1, we illustrate the structure of the virtual experiment system.

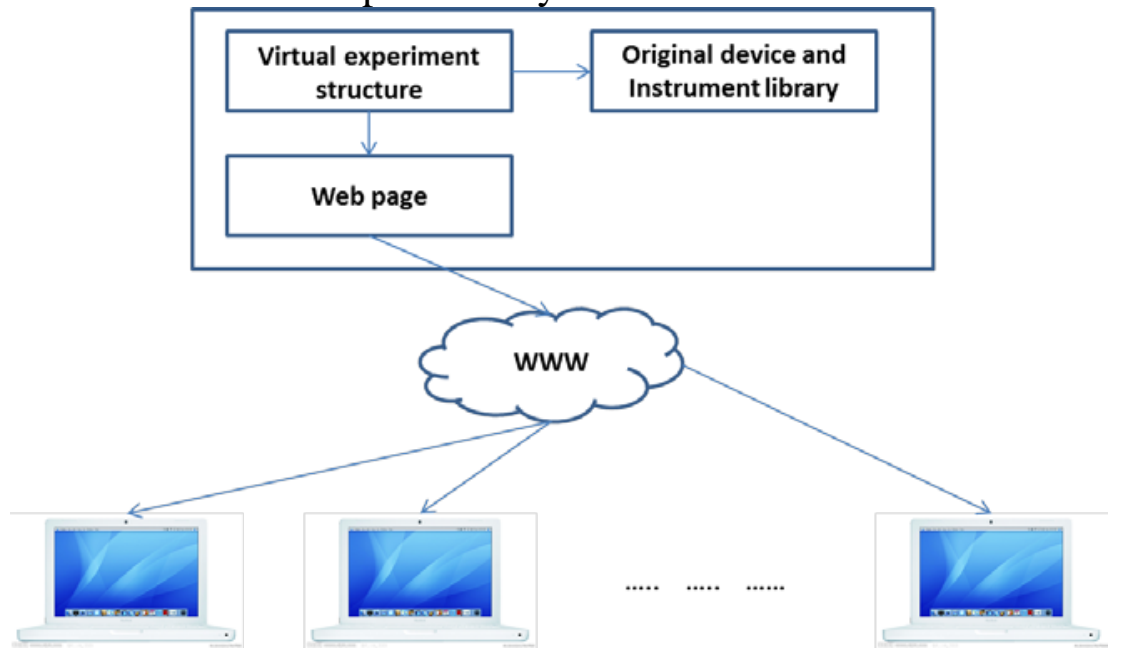

Fig. 1The General Structure of the Virtual Experiment System

Therefore, we conduct research on the design and implementation of computer network virtual laboratory based on ASP.NET. The detailed discussion will be introduced in the following sections.

\section{The Proposed System}

The Principles of Virtual Laboratory. Experimental operation is the basic unit of components and these components can be viewed as a virtual entity. Each virtual entity is a relatively independent function which can carry on the behavior and the environment relations unit. We need to construct the experiment in the virtual experiment of component model which uses mathematical model to replace the physical existence of the specific physical device and the mathematical model should be able to correctly reflect the physical and chemical characteristics of the device which should be convenient for numerical calculation on the computer [4-5]. Components of their work are the foundation of further work that directly affects the precision of the whole process of experiment and calculation speed. No virtual experiment instrument and conventional control panel is considered, so with the aid of computer graphics environment, on a computer screen to build graphical soft panel to replace the experiment instrument and conventional control panel. Soft panel has a similar to the actual experiment and the actual machine knobs, switches, indicator lights and other control components. Users through the mouse or keyboard could soft panel and virtual components can be like in the traditional laboratory experiments. Rely on it by experiments of simulation program, experimental units, tools and resources. Users can add new objects, the establishment of the new experiment and translate them into hypertext files to expand laboratory. These two kinds of definitions on different direction, but points out that the nature of the virtual laboratory is a laboratory, without the wall through the computer network system of remote control and interactive system, researchers or students will not be restricted by space-time, virtual experiment operating anytime and anywhere, and share the instruments and equipment, Shared data and computer resources, collaboration or get remote guidance, etc. Complete function of virtual laboratory is the core of virtual instrument and it is a system with general computer as the controller by the software to realize the human-computer interaction. Network virtual laboratory is a virtual instrument technology, the combination of the computer technology and network technology and the main function of networked virtual instrument. Networked virtual instrument of virtual instrument function decomposition and then connect these 
functions through the network to form a network of virtual instrument system [6-7]. The detailed structure and pattern is shown below in the figure 2 .

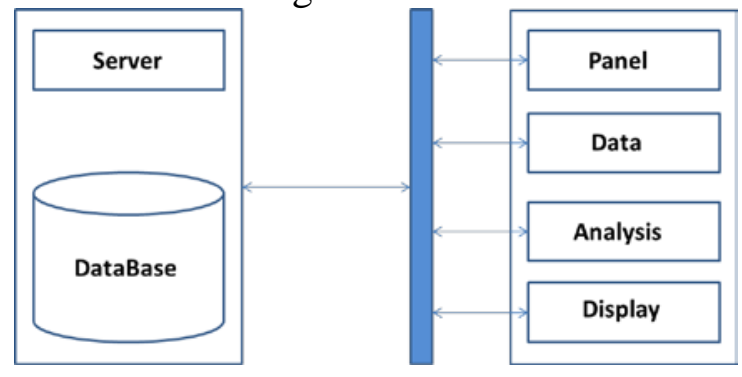

Fig. 2The General Structure and Pattern of the Virtual Instrument System

The Concepts of ASP.NET Technique. At early time the web server sends the basic static page, accessing to web with HTML format. The disadvantage is that after completion of the static page editor, it will cause the content of the page not having dynamic changes. ASP combines the components of HTML, Script and ActiveX together, which can form the application running on the server. In order to reduce the entry intensity of equipment downtime information, improve the efficiency and accuracy, this article make the usual repetitive equipment downtime information such as product line, process, equipment, product information and related constants data merge together to form a comprehensive downtime information. Based on WinCE, Windows XP embedded devices can achieve real-time processing, a data acquisition terminal. An associated unit according to the requirements of open architecture design experimental electric circuit, in addition to further standardize the principle can be composed of computer architecture, also provides ample for experimental teaching software and hardware design space design space. Combined with Sky-river Server, such as SQL Server software, data management Server component Server components, signal analysis, expert system web Services that can build networked virtual instrument, such as the Server. PC local resources can be fully used to realize fast interactive operation of desktop virtual instrument and virtual instrument based on web and portable virtual instrument, a networked virtual instrument to the client. Achieved through the method of the software, because of the complexity and diversity of network experiment requires the user to write a lot of code, and the vast majority of grammatical structure is complex, the network simulation software is difficult to grasp. With hardware method, need a lot of equipment, the cost is large, and provided experiment is limited, generally limited to local area network (LAN) and lower network experiment, remote interactive performance is not good, can't completely solve the problem of computer network remote experiment teaching.

The User-Interface and Combination. In the following figure 1, we illustrate the basic user interface and the corresponding pattern for the system. The Model-View-Controller (MVC) design pattern which was used to develop the application in ASP.NET is similar to the MVC design pattern for Java with the main difference being that the view was developed using an Active Server Pages (ASP) and the controller is in C\# code. The views were developed using ASP.NET web pages. In this architecture, the remote client through the browser to access Web pages on the server, and in the Web pages in the virtual experiment are embedded soft component. The server through the virtual experiment of soft component for experimental environment and various kinds of experimental simulation, and experimental operation request received from the client. According to the client's operation, the virtual experiment of soft component adjustment state of virtual components, simulation experiment phenomenon, output corresponding. So-called visualization mapping refers to the system of human-machine interface and system of establish the contact between calculation module and it is a key step to realize human-computer interaction. Visualization mapping is a two-way system, namely on the one hand, the graphics and animation in the form of the screen mapping as required for the simulation of the system and the data. On the other hand, it will be transformed into digital information system simulation calculation of the intuitive, using pictures or graphical representation of the change with time and space of physical phenomenon or physical quantities, and presents in front of the experimenter. There are two engines for developing the views, namely razor (*.cshtml) and aspx (*.aspx). The razor engine was used to develop all the views, the 
controllers were developed using C\# classes (*.cs) and the models were developed using Entity Classes which are also C\# classes (*.cs). The detailed is shown in the figure 3.

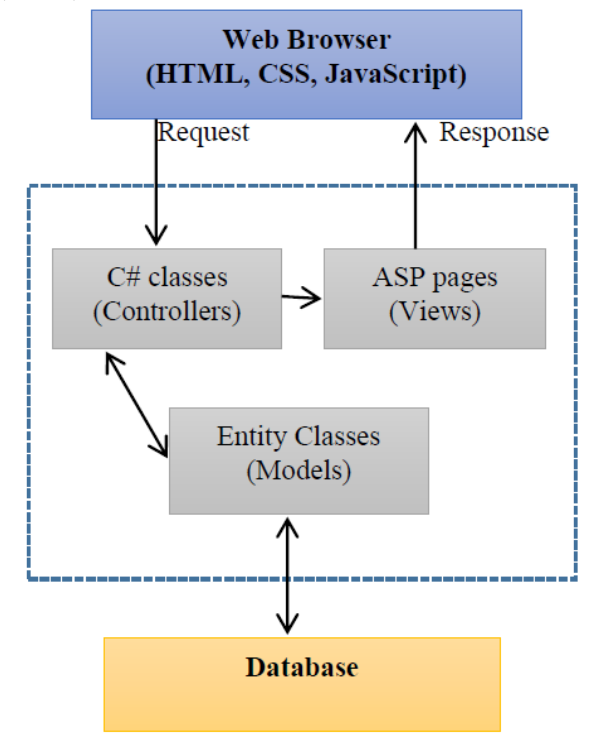

Fig. 3The User-Interface and the Corresponding Structure

\section{Experiment and Simulation}

In the figure 4, we show the experimental result on the efficiency of different coding languages among PHP, Java and ASP.NET. The result is evident and ASP.NET performs well.

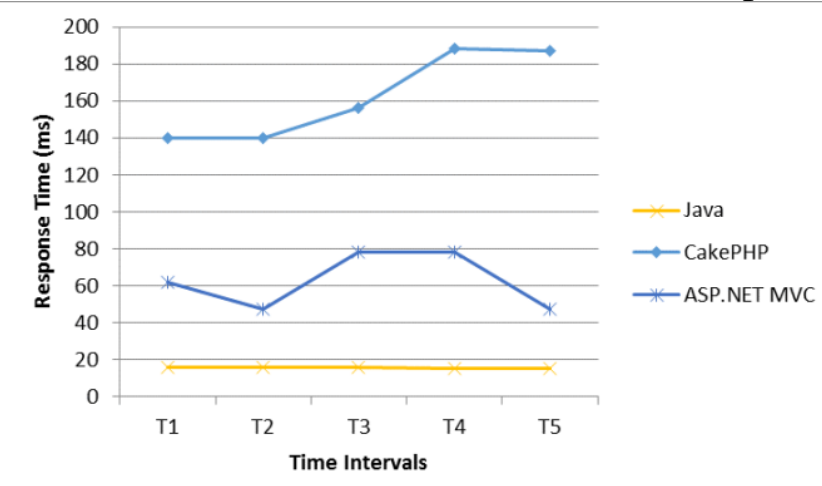

Fig. 4The Simulation Result for the Proposed Approach

\section{Conclusion and Summary}

Along with the development of computer technology, network experimental simulation teaching become an important research topic in the field of education, through the establishment of network experimental simulation system can effectively solve the problem of experimental teaching in the network education. This paper first on the present situation of computer network experiment simulation system were analyzed, the main is about computer network experiment simulation system is introduced in this paper the research achievements in the process, so as to further grasp and discuss hot topics in the study of computer network experiment simulation system problems and the direction. Aiming at some disadvantages of the existing network experiment teaching, research and design realization of computer network experiment simulation system based on ASP.NET is a good way. The classification of the knowledge of the computer network, computer network experiment simulation teaching research and ASP.NET technology on the basis of analysis of the application of research, based on the design of computer network experiment simulation system of ASP.NET, then elaborated the related research and development of the system the discussion of teaching model, system structure analysis and the introduction of system function module, etc. In this paper, a 
concrete analysis and presented, including the development of computer network experiment simulation system based on ASP.NET environment, development language and platform design, database design, system model, function modules and implementation of the system and so on key question solution technique problems.

\section{References}

[1] Xu L, Huang D, Tsai W. Cloud-Based Virtual Laboratory for Network Security Education[J]. Education IEEE Transactions on, 2014, 57(3):145 - 150.

[2] Aydogmus Z, Aydogmus O. A web-based remote access laboratory using SCADA[J]. Education, IEEE Transactions on, 2009, 52(1): 126-132.

[3] Rui Z. Computer teaching based on X3D virtual network laboratory research[J]. Network Security Technology \& Application, 2014.

[4] Zhanpeng H, Shizhong J, Jinyu L. Realization of digital image processing virtual laboratory based on Web [J]. Experimental Technology and Management, 2010, 12: 038.

[5] Luo G, Qian Z, Dong M, et al. Lecture Notes in Computer Science[M]. Springer International Publishing, 2014.

[6] Xiao-hui W. Study on the design of Network Virtual Laboratory[J]. Journal of Shenyang Institute of Engineering, 2014.

[7] Xie W, Yang X, Li F. A virtual laboratory platform and simulation software based on web[C]//Control, Automation, Robotics and Vision, 2008. ICARCV 2008. 10th International Conference on. IEEE, 2008: 1650-1654. 